\title{
Gestão de recursos humanos e manufatura enxuta: evidências empíricas do setor automotivo brasileiro
}

\author{
Wesley Ricardo de Souza Freitas ${ }^{\text {a* }}$, Charbel José Chiappetta Jabbour ${ }^{\text {b }}$ \\ Adriano Alves Teixeirac ${ }^{c}$, Ana Beatriz Lopes de Sousa Jabbour ${ }^{\mathrm{d}}$ \\ a*wesley007adm@yahoo.com.br, UFMS, Brasil \\ bprof.charbel@gmail.com.br, UNESP-Bauru, Brasil \\ caatadrianobirigui@gmail.com, FEA-RP/USP, Brasil \\ dablsjabbour@gmail.com, UNESP-Bauru, Brasil
}

\begin{abstract}
Resumo
0 objetivo deste artigo é verificar se as práticas de gestão de recursos humanos estão relacionadas positivamente com a adoção de práticas de manufatura enxuta em empresas brasileiras do setor automotivo. Para tanto, foi proposto um framework relacionando esses conceitos com a proposição de uma hipótese de pesquisa. Os dados coletados de 75 empresas foram analisados por meio de modelagem de equações estruturais. 0 principal resultado desta pesquisa é que, de fato, a gestão de recursos humanos vem se relacionando de forma positiva com a manufatura enxuta nas empresas analisadas, mas tal relação tende a ocorrer de maneira fraca-moderada.
\end{abstract}

Palavras-chave

Gestão de recursos humanos. Manufatura enxuta. Setor automotivo. Brasil.

\section{Introdução}

Evidencia-se na literatura acadêmica que a gestão de recursos humanos (GRH ou RH) contribui para a melhoria da performance e implementação de práticas de gestão organizacional (SCHULER; JACKSON, 1987; JANSSENS; STEYAER, 2009; PAAUWE, 2009; FERGUSON; REIO JUNIOR, 2010). Adicionalmente, Boudreau et al. (2003) afirmam que a gestão de recursos humanos é tão importante para o bom desempenho das organizações manufatureiras quanto as melhorias e atualizações tecnológicas por elas adotadas. Diante desse panorama, desenvolver práticas de GRH congruentes com esse cenário competitivo torna-se necessário e desafiador para os gestores empresariais.

Por outro lado uma das mais importantes abordagens de gestão da produção nesse ambiente competitivo é o sistema denominado manufatura enxuta, que consiste na adequação do sistema produtivo em termos de redução de custo (OHNO, 1988), produção sem defeitos (WOMACK; JONES; R0OS, 1990) e orientada para as necessidades dos clientes (DENNIS, 2008). Mas estariam as práticas de recursos humanos alinhadas às novas abordagens gerenciais apoiando a implementação da manufatura enxuta?

Frente a essa conjuntura, estabelece-se a questão central da pesquisa deste artigo: As práticas de gestão de recursos humanos influenciam positivamente a adoção de práticas de manufatura enxuta em empresas brasileiras do setor automotivo?

Como consequência, o objetivo deste artigo é verificar se as práticas de gestão de recursos humanos estão influenciando positivamente a adoção de práticas de manufatura enxuta em empresas brasileiras do setor automotivo - com foco no segmento de autopeças e componentes automotivos.

0 setor automotivo brasileiro teve seu início nos anos 1950. Atualmente, são 26 montadoras com 53 fábricas abastecidas por mais de 600 empresas de autopeças e com uma capacidade produtiva instalada de 4,3 milhões de veículos e 109 mil máquinas agrícolas por ano, o que posiciona o 
Brasil entre os seis maiores produtores de veículos do mundo (ASSOCIAÇÃO..., 2011). O setor emprega aproximadamente 1,5 milhão de pessoas, fatura anualmente mais de US\$ 107,6 bilhões (incluindo autopeças) e tem uma participação direta no produto interno bruto (PIB) brasileiro de 5,2\%, podendo-se chegar a $22,5 \%$ do PIB se considerados os efeitos indiretos (ASSOCIAÇÃO..., 2011).

Para relatar a pesquisa conduzida, este artigo está estruturado da seguinte forma: na seção 2, como pano de fundo, apresentam-se os fundamentos conceituais sobre manufatura enxuta e gestão de recursos humanos e destacam-se o framework e a hipótese de pesquisa; na seção 3 apresentam-se os procedimentos metodológicos; na seção 4 são apresentados os resultados da pesquisa empírica e na seção 5 discute-se a proposta do trabalho à luz do referencial teórico, bem como apresentam-se as considerações finais deste estudo.

\section{Fundamentos conceituais, framework e hipótese de pesquisa}

\subsection{Manufatura enxuta}

Na década de 1990, a partir da publicação do livro: A máquina que mudou o mundo (WOMACK; JONES; ROOS, 1990), a expressão manufatura enxuta ficou conhecida como sinônimo das práticas pioneiras da Toyota - Sistema Toyota de Produção (SCHONBERGER, 2007) e começou a tornar-se uma importante prática de gestão competitiva da produção.

Apesar do Sistema Toyota de Produção ser discutido desde a década de 1950, em função de sua evolução, a partir das incorporações e das adaptações ocidentais, o termo manufatura enxuta ainda não possui singularidade de entendimento e medição (SHAH; WARD, 2007; PETTERSEN, 2009). Por exemplo, Womack, Jones e Roos (1990), resumidamente, afirmam que manufatura enxuta significa fazer mais com menos. Já Shah e Ward (2007) definem manufatura enxuta como um sistema sociotécnico integrado cujo objetivo é eliminar desperdícios pela concomitante redução ou minimização da variabilidade interna de fornecedores e de clientes. Para ScherrerRathje, Boyle e Deflorin (2009), manufatura enxuta é uma filosofia focada em identificar e eliminar desperdícios em toda a cadeia de valor, não somente dentro da organização. Como consequência da abordagem multidimensional da manufatura enxuta, há uma grande variedade de práticas de gestão e metas que a constituem (SHAH; WARD, 2003).

Nesse sentido, estão dentre os principais objetivos e metas da manufatura enxuta: diminuição de custo (OHNO, 1988); fabricação de produtos sem defeitos, conforme a necessidade dos clientes (WOMACK; JONES; ROOS, 1990); e foco no cliente (DENNIS, 2008). Sendo assim, para que seja possivel atingir os objetivos relatados, várias práticas podem ser estabelecidas, como por exemplo, as apresentadas nos trabalhos de Biazzo e Panizzolo (2000), Shah e Ward (2003), Bhasin e Burcher (2006) e Pettersen (2009). 0 Quadro 1 busca sistematizar essas principais práticas e características associadas à manufatura enxuta.

Todavia, para que essas práticas da manufatura enxuta sejam efetivas, necessitam do apoio de várias práticas de gestão de recursos humanos, conforme explanação a seguir.

\subsection{Gestão de recursos humanos}

Para Fischer (2002), gestão de pessoas diz respeito a como a empresa organiza, gerencia e orienta o comportamento das pessoas. Pode ser entendida, ainda, como um conjunto de atividades executadas

Quadro 1. Práticas e características associadas à manufatura enxuta.

\begin{tabular}{|l|l|}
\hline \multicolumn{1}{|c|}{ Práticas e caracteristicas } & \multicolumn{1}{c|}{ Descrição } \\
\hline $\begin{array}{l}\text { LM1 - Funcionário multifuncional/ envolvimento } \\
\text { no processo }\end{array}$ & $\begin{array}{l}\text { Desenvolvimento das habilidades dos funcionários e estímulo à autonomia para evitar } \\
\text { falhas ao longo do processo. }\end{array}$ \\
\hline LM2 - Melhoria contínua & Busca a continua melhoria incremental em qualidade, custo, entrega e projeto. \\
\hline LM3 - 5S & $\begin{array}{l}\text { Uma forma de gestão visual destinada a reduzir a desordem e a ineficiência no } \\
\text { ambiente produtivo e administrativo. }\end{array}$ \\
\hline LM4 - Manutenção produtiva total & $\begin{array}{l}\text { 0 objetivo é melhorar a confiabilidade e a capacidade das máquinas por meio de } \\
\text { regimes periódicos de manutenção. }\end{array}$ \\
\hline LM5 - Kanban & Sistema de cartões para criar um fluxo puxado. \\
\hline LM6 - Just in time & Busca o fluxo contínuo da produção. \\
\hline LM7 - Redução de lote/redução de estoque & $\begin{array}{l}\text { Formação de pequenos lotes de produção para reduzir o estoque em processo e } \\
\text { aumentar a variedade. }\end{array}$ \\
\hline LM8 - Círculos de melhoria/círculos kaizen & $\begin{array}{l}\text { Promoção de discussões sistemáticas entre operadores e gestores a fim de promover a } \\
\text { melhoria incremental contínua. }\end{array}$ \\
\hline LM9 - Desenvolvimento/ colaboração de fornecedor & $\begin{array}{l}\text { Atividades voltadas a desenvolver relacionamento com fornecedor, a fim de obter sua } \\
\text { colaboração. }\end{array}$ \\
\hline
\end{tabular}


em prol da adequada gestão da força de trabalho (BOSELIE; DIETZ; BOON, 2005). Portanto, a gestão de pessoas é

uma série de decisões integradas que formam as relações de trabalho; sua qualidade influencia diretamente a capacidade de a organização e seus agentes atingirem seus objetivos (MILKOVICH; BOUDREAU, 2006, p. 19).

Em meados de 1990, visando contrapor argumentos de que a área de recursos humanos (RH) pouco contribui com as organizações, houve um grande número de pesquisas apresentando a correlação positiva entre os RH e o desempenho das organizações (LENGNICK-HALL et al., 2009). Entretanto, recentemente, a literatura especializada argumenta que essa relação tende a ser influenciada por fatores culturais, econômicos, políticos e sociais dos países em que as organizações se inserem (DEWETTINCK; REMUE, 2011). Assim, a eficácia organizacional não pode ser garantida pela simples existência de práticas de $\mathrm{RH}$, sendo essa contingencial (BOSELIE; DIETZ; BOON, 2005), ou seja, é influenciada por fatores externos. Portanto, para Lengnick-Hall et al. (2009), o principal desafio da área de RH é alinhar as suas principais práticas (Quadro 2) à estratégia e desempenho das organizações.

\subsection{Suporte da gestão de recursos humanos para a manufatura enxuta}

Práticas de recursos humanos como participação dos funcionários em programas de melhoria contínua; equipes multifuncionais; treinamento de funcionários; e rodízio de funções são reconhecidas por formar o núcleo de um programa de manufatura enxuta (SHAH; WARD, 2003; FARRIS et al., 2009). Em consonância com as exigências do modelo de manufatura enxuta, geralmente o sistema de trabalho é caracterizado por: busca de flexibilidade da força de trabalho; descentralização da tomada de decisão; ênfase no trabalho em equipe e estrutura de emprego (por exemplo, garantias de segurança, formação continuada, remuneração baseada em sistemas de desempenho etc.), visando assegurar que a força de trabalho seja altamente motivada e comprometida (BIAZZO; PANIZZOLO, 2000). Além dessas questões, Doolen e Hacker (2005) adicionam a avaliação de desempenho dos funcionários e o sistema formal de recompensa como elementos que caracterizam o sistema de trabalho no ambiente de manufatura enxuta.

Menezes, Wood e Gelade (2010) consideram que a melhoria contínua, uma das características da manufatura enxuta, não pode ser alcançada sem a participação dos funcionários. Por exemplo, aprendizagem e cooperação facilitam o processo de gestão e a integração ao longo do tempo, fazendo com que as organizações que adotam essa filosofia gerencial superem aquelas que não o fazem.

Martín e García (2010) comprovaram que práticas de gestão de recursos humanos estão associadas com a introdução de manufatura enxuta e com os resultados organizacionais obtidos. As empresas que adotam a maioria das práticas de manufatura enxuta são também aquelas que tomam o cuidado de treinar os trabalhadores na utilização dessas práticas. A combinação da manufatura enxuta com as práticas de gestão de recursos humanos ajuda a reduzir o lead time e o estoque e a aumentar a produtividade.

0 evento kaizen, uma das práticas da manufatura enxuta, funciona como um mecanismo de melhoria cada vez mais comum nas organizações, visando a transformação da área de trabalho e o desenvolvimento de funcionários (FARRIS et al., 2009). Segundo Doolen et al. (2008), o apoio da gestão mostra-se positivo na conclusão de um evento kaizen. Além disso, a equipe do evento kaizen, com um escopo

Quadro 2. Práticas de GRH.

\begin{tabular}{|l|l|}
\hline \multicolumn{1}{|c|}{ Práticas } & \multicolumn{1}{c|}{ Conceitos } \\
\hline $\begin{array}{l}\text { HR1 - Recrutamento } \\
\text { e seleção }\end{array}$ & $\begin{array}{l}\text { Recrutamento consiste no processo de atração de novas pessoas para os quadros da empresa; e a seleção consiste } \\
\text { na escolha das pessoas certas, separando os não adequados antes de "entrarem pela porta, não depois" (DESSLER, } \\
\text { 2003, p. 106). As empresas podem buscar trabalhadores no mercado interno ou podem recorrer ao mercado externo } \\
\text { (SCHULER; JACKSON, 1987). Assim, segundo Santos (1973, apud FRANÇA; ARELLANO, 2002), nem sempre se } \\
\text { escolhe os melhores candidatos, mas sim os mais adequados às expectativas organizacionais. }\end{array}$ \\
\hline HR2 - Treinamento & $\begin{array}{l}\text { É uma ação organizacional planejada, que possibilita a aquisição de habilidades técnicas e comportamentais que } \\
\text { podem tornar o indivíduo mais apto a desempenhar suas funços atuais ou futuras (BORGES-ANDRADE, 2002). }\end{array}$ \\
\hline $\begin{array}{l}\text { HR3 - Avaliação de } \\
\text { desempenho }\end{array}$ & $\begin{array}{l}\text { As avaliações podem ser definidas como um processo que visa determinar os resultados do trabalho de um } \\
\text { funcionário (TÜRK; ROOLAHT, 2007). Avaliação de desempenho é um processo formalizado para classificar o } \\
\text { desempenho atual do funcionário (STONER; FREEMAN, 1999). }\end{array}$ \\
\hline HR4 - Recompensas & $\begin{array}{l}\text { 0 termo refere-se a "todos os pagamentos monetários e todos os bens ou mercadorias usados para recompensar } \\
\text { empregados" (DAFT, 1999, p. 263). }\end{array}$ \\
\hline HR5 - Benefícios & $\begin{array}{l}\text { São facilidades e conveniências compartilhadas pela organização e pelos funcionários que não estão contempladas } \\
\text { no salário direto mas têm impactos financeiros no “bolso" dos funcionários e no "caixa” da organização" (OLIVEIRA; } \\
\text { LEONE, 2008, p. 108). }\end{array}$ \\
\hline
\end{tabular}


mais limitado, é mais capaz de cumprir os objetivos específicos de negócio. Em outros termos, tendo o suporte de um líder (facilitador) e incentivador, cada grupo de funcionários é capaz de ser estimulado a discutir e solucionar problemas partindo da autonomia, treinamento e delimitação do impacto positivo daqueles resultados em seus interesses próprios e organizacionais.

No entanto, Chen, Lindeke e Wyrick (2010) fazem algumas considerações sobre o efeito adverso do modelo de manufatura enxuta nos aspectos de recursos humanos. Segundo esses autores, a multifuncionalidade acarreta em carga elevada de trabalho e estresse, reduzindo a capacidade de inovar dos funcionários. Essa exigência quanto às habilidades e à flexibilidade dos colaboradores também gera um clima de insegurança no emprego, pois há o receio de não serem capazes de se manter na empresa. Portanto, essas questões merecem análises mais profundas, através de estudos empíricos.

\subsection{Framework e hipótese de pesquisa}

Na Figura 1 é apresentado o framework da pesquisa com os relacionamentos entre os construtos de recursos humanos (HR) e de manufatura enxuta (LM). Com base nos conceitos apresentados na seção 2, a hipótese de pesquisa é: $\mathrm{H}_{1}$ - As práticas de gestão de recursos humanos se relacionam positivamente com a adoção de práticas de manufatura enxuta.

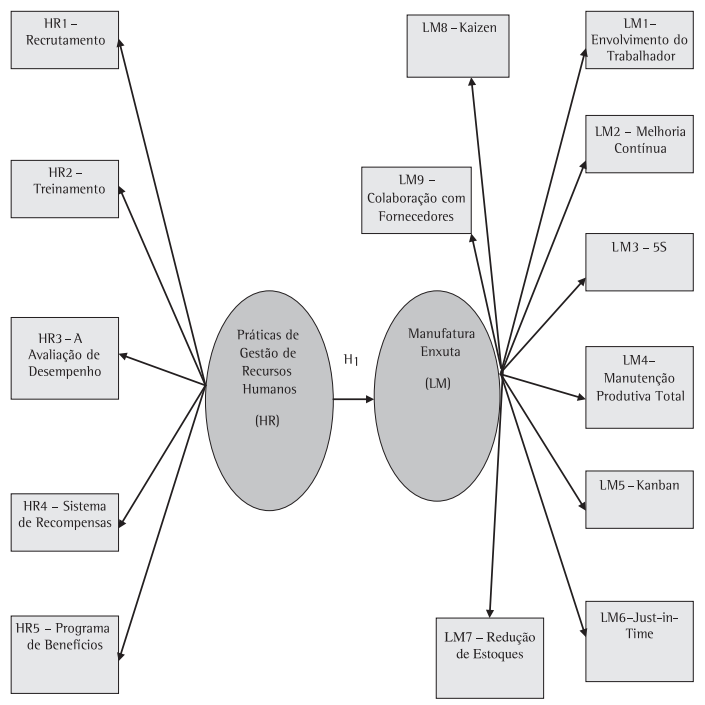

Figura 1. Framework relacional da hipótese da pesquisa.
Os testes do framework e da hipótese de pesquisa seguiram os procedimentos metodológicos descritos a seguir.

\section{Metodologia}

Esta pesquisa é quantitativa, baseada na estratégia survey, com a finalidade de teste de hipótese para a confirmação ou refutação do arcabouço teórico previamente revisado. 0 setor alvo desta pesquisa é o setor automotivo brasileiro, em específico o segmento de autopeças, cujas características foram apresentadas na seção 1 .

Para a coleta de dados utilizou-se como instrumento um questionário estruturado sobre os conceitos previamente revisados nas seções 2.1, 2.2 e 2.3, elaborado segundo as recomendações em Synodinos (2003).

0 questionário possui, além de informações sobre a caracterização das empresas respondentes, dois blocos de assertivas: um para o construto gestão de recursos humanos e outro para manufatura enxuta. No total, o questionário (Apêndices A e B) apresenta cinco assertivas sobre gestão de recursos humanos e nove assertivas sobre manufatura enxuta. A primeira versão do questionário foi submetida à validação de conteúdo por meio da análise de cinco pesquisadores na área, além de adequação aos pressupostos conceituais. 0 questionário, em sua versão final, foi hospedado em um ambiente virtual especialmente elaborado para esta pesquisa. Adotou-se uma escala Likert de cinco pontos, onde 1 representa discordo totalmente e 5 concordo totalmente, para a mensuração das práticas de recursos humanos; uma escala likert cinco pontos foi adotada para as práticas de manufatura enxuta com os extremos não implementado e completamente implementado.

Os dados desta pesquisa foram coletados entre os meses de outubro de 2010 e março de 2011. Primeiramente foram coletados endereços de e-mails e informações telefônicas, junto ao Sindicato Nacional da Indústria de Componentes para Veículos Automotores - SINDIPEÇAS, de 654 empresas do setor automotivo (segmento de autopeças) localizadas no Brasil. Foram obtidas informações sobre o nome, cargos e contato de diretores ou gerentes de produção. Acredita-se que esses respondentes têm as condições necessárias para responder o questionário de pesquisa, uma vez que são, geralmente, responsáveis tanto pela implantação de manufatura enxuta quanto pela gestão de recursos humanos na área em que atuam. Foram enviados e-mails para esse conjunto de respondentes e respectivas empresas com uma breve explicação sobre a pesquisa e um convite para participação direcionado ao gestor de produção. No 
e-mail havia um link para encaminhar o respondentealvo diretamente para o questionário hospedado no ambiente virtual da pesquisa. Também foram realizadas ligações telefônicas visando aumentar o percentual de retorno de questionários válidos, com as quais se buscou contatar o funcionário responsável pela área de produção da empresa. Assim, foram recebidos 72 questionários, coletados por meio do site da pesquisa, e mais 4 questionários coletados por meios alternativos, conforme solicitado pelos respondentes. Deste modo, foram obtidos 76 questionários, dos quais se desconsiderou um, por apresentar dados incompletos. A taxa de retorno foi de $11,11 \%$ ou 75 questionários válidos.

Tanto a taxa de retorno quanto a quantidade de questionários obtidos podem ser consideradas adequadas, pois: (a) há pesquisas que indicam taxas de retorno menores do que 10\% como válidas na área de gestão de operações/produção (SYNODINOS, 2003; MURILLO-LUNA; GARCÉS-AYERBE; RIVERA-TORRES, 2011); (b) o modelo conceitual proposto possui 14 variáveis, o que exige, segundo a regra de Hair Junior et al. (2005), minimamente, 70 questionários válidos; (c) o Goodness-of-Fit (GoF), que avalia a validade global do modelo estatístico final, deve ser maior do que 0,36 (WETZELS; ODEKERKENSCHRODER; VAN OPPEN, 2009) e de fato foi, conforme a seção 4 relata. Sobre o perfil da amostra, segundo a proposta de classificação de empresas por quantidade de funcionários recomendada pelo Sebrae, dos 75 questionários obtidos, 18,66\% foram de pequenas empresas, 54,67\%, de médias empresas e $26,67 \%$, de grandes empresas. Cada questionário preenchido alimentou automaticamente uma planilha de dados para posterior processamento estatístico.

0 framework de pesquisa (apresentado na seção 2.4) guiou o processo de análise de dados, que envolveu o uso de procedimentos estatísticos com o suporte de planilhas de dados, do software Statistical Package for Social Sciences (SPSS) da IBM (versão 19.0) (apoio a análise univariada e bivariada das variáveis), e do software Smart PLS 2.0 (apoio a análise de modelagem de equações estruturais).

Primeiramente, para a aplicação de modelagem de equações estruturais (MEE), optou-se por aplicar análise de componentes principais (ACP) (redução de dados), por meio do método varimax para cada construto. A aplicação dessa análise envolve a verificação de medidas de adequação da amostra, como teste Kaiser-MeyerOlkin - KMO - que verifica o valor da correlação entre as variáveis - e teste Bartlett - que testa a hipótese de a matriz de correlação ser a matriz identidade - para aplicação de técnicas multivariadas (MEE). Se os valores forem compatíveis com os parâmetros da literatura, analisam-se cada um dos fatores formados.
Isto ocorre pela análise do valor do Alfa de Cronbach (mede a confiabilidade dos construtos), variância média explicada e autovalor (o quanto de variância da nuvem de dados é absorvido por ele). Atendendo aos parâmetros da literatura (acima de 0,7 para o Alfa de Cronbach, acima de 1 para autovalor e acima de 0,6 de variância média explicada), começa-se a analisar cada variável. Isto se concretiza pela análise da diagonal principal da Matriz Anti-Imagem (que deve apresentar valores acima de 0,6), comunalidade (explica a aderência de uma dada variável aos diversos fatores obtidos) e carga fatorial. Como subprodutos dessa análise, obtêm-se as correlações, as médias e outras medidas estatísticas (essas informaç̧ões foram obtidas com o SPSS). Após esses procedimentos, os fatores obtidos e purificados (sem variáveis com medidas estatísticas comprometidas) são inseridos no SmartPLS. Nesse software podem-se obter os testes empíricos do framework conceitual proposto. Primeiramente, devem-se obter os coeficientes entre variáveis e entre os construtos. Na sequência, deve-se verificar se esses coeficientes são estatisticamente válidos por meio do $\mathrm{p}$ value obtido. Finalmente, deve-se obter o valor do GoF (Goodness-of-Fit) que, por meio da média geométrica entre as médias da variância explicada e do R2 das variáveis exógenas, é avaliado de forma global, pontuando o ajuste estatístico do modelo.

A seção 4 apresenta, detalhadamente, os procedimentos estatísticos associados a cada um dos resultados obtidos.

\section{Resultados}

A redução dos dados de todas as variáveis, tanto do construto práticas de gestão de recursos humanos (HR) quanto do construto manufatura enxuta (LM), foi realizada usando a análise de componentes principais, pelo método varimax. Esse procedimento foi realizado independentemente para cada um dos construtos. Nesse primeiro procedimento, com base nos valores de referência indicados por Costa, Souza e Silva (2008), Hair Junior et al. (2005), Hair Junior, Ringle e Sarstedt (2011) e Wetzels, Odekerken-Schroder e Van Oppen (2009), os principais indicadores de qualidade estatística foram:

- Adequação da amostra para cada fator individual pelo teste KMO (Kaiser-Meyer-Olkin);

- Teste Bartlett de esfericidade;

- 0 Eigenvalue (autovalor) para cada fator, dos quais foram extraídos os fatores com valores iguais ou superiores a 1,0 ;

- A variância acumulada explicada;

- Cálculo do Alfa de Cronbach para cada fator; 
- Diagonal principal da Matriz Anti-Imagem;

- Comunalidades das variáveis.

A fim de refinar os resultados, a análise de componentes principais passou a revelar apenas cargas de variáveis acima de 0,6, fatores com autovalores maior que 1 e coeficientes da diagonal da Matriz Anti-lmagem maior do que 0,6. Verificou-se se a comunalidade das variáveis é igual ou superior a 0,5 para cada variável (HAIR JUNIOR et al., 2005) de cada construto.

Em relação ao construto práticas de gestão de recursos humanos (HR), apenas um fator foi formado, explicando uma variância acumulada aproximada de 68,12\%, com autovalor de 2,72 e valores adequados na diagonal principal da Matriz Anti-Imagem $(0,71 ; 0,61 ; 0,68 ; 0,63)$. 0 teste KMO, que verifica a adequação da amostra, foi de 0,662 , considerado adequado, assim como o valor obtido com o teste Bartlett de esfericidade (141,41 - com significância 0,000$)$ e pelo Alfa de Conbrach $(0,84)$. 0 construto práticas de gestão de recursos humanos (HR) passou a ser formado pelas variáveis HR1, HR2, HR3 e HR4 (Tabela 1). A variável HR5 foi excluída por apresentar baixa comunalidade $(0,38)$. 0 Apêndice C apresenta medidas estatísticas complementares para o construto HR.

Em relação ao construto manufatura enxuta (LM), apenas um fator foi formado, explicando uma variância acumulada aproximada de 64,27\%, com autovalor de 5,78 e valores adequados na diagonal principal da Matriz Anti-lmagem (0,917; 0,904; 0,927; 0,903; 0,867; 0,841;0,891;0,943;0,908). 0 teste KMO foi de 0,900, considerado adequado, assim como o valor obtido com o teste Bartlett de esfericidade $(460,202$ - com significância 0,000) e pelo Alfa de Conbrach $(0,927)$. Todas as variáveis do construto LM apresentaram valores satisfatórios (Tabela 2). Medidas estatísticas complementares para o construto LM podem ser verificadas no Apêndice D.

Em seguida, a modelagem de equações struturais (structural equation modeling) Partial Least Squares (SEM-PLS) foi utilizada. A modelagem de equações estruturais por meio de PLS é considerada uma análise multivariada de segunda geração. Ela é especialmente útil quando se trabalha com teoria complexa (relacionando conceitos tradicionais, como recursos humanos e manufatura enxuta) ou em estágios iniciais de desenvolvimento. Um modelo estrutural foi criado contendo os construtos obtidos a partir da análise de componentes principais, como explicado acima. As análises foram realizadas usando o software SmartPLS 2.0 (SOSIK; KAHAl; PIOVOSO, 2009).

Indicadores de boa qualidade para o modelo proposto foram alcançados em termos de variância média extraída (validade convergente), confiabilidade composta, Alfa de Cronbach e comunalidades, tanto para o construto RH, quanto para LM. Para checar a confiabilidade (que apura a precisão com que 0 construto mede exatamente aquilo que realmente deveria medir) e a validade satisfatória (que testa a relação de uma variável com outra variável de um mesmo construto), o valor de confiabilidade composta deve ser superior a 0,7, enquanto o valor da validade convergente deve ser superior a 0,5 (FOLTZ, 2008). Na Figura 2 são apresentados os coeficientes de correlação entre variáveis e entre construtos.

Na Figura 2 há duas importantes informações. A primeira é quanto à relação positiva entre os construtos HR e LM, tendo em vista o valor de 0,531. A segunda informação é que, com base em Hair Junior, Ringle e Sarstedt (2011), o valor de R2 pode ser próximo a 0,75, 0,50 e 0,25 sendo considerado, respectivamente:

Tabela 1. Resultado da análise de componentes principais para HR.

\begin{tabular}{ccc}
\hline Variáveis & Carga & Comunalidades \\
\hline HR1 & 0,85 & 0,72 \\
HR2 & 0,86 & 0,74 \\
HR3 & 0,87 & 0,76 \\
HR4 & 0,70 & 0,50 \\
\hline
\end{tabular}

Tabela 2. Resultado da análise de componentes principais para LM.

\begin{tabular}{ccc}
\hline Variáveis & Carga & Comunalidades \\
\hline LM1 & 0,79 & 0,63 \\
LM2 & 0,84 & 0,71 \\
LM3 & 0,81 & 0,65 \\
LM4 & 0,81 & 0,66 \\
LM5 & 0,74 & 0,54 \\
LM6 & 0,81 & 0,65 \\
LM7 & 0,82 & 0,67 \\
LM8 & 0,82 & 0,68 \\
LM9 & 0,75 & 0,57 \\
\hline
\end{tabular}

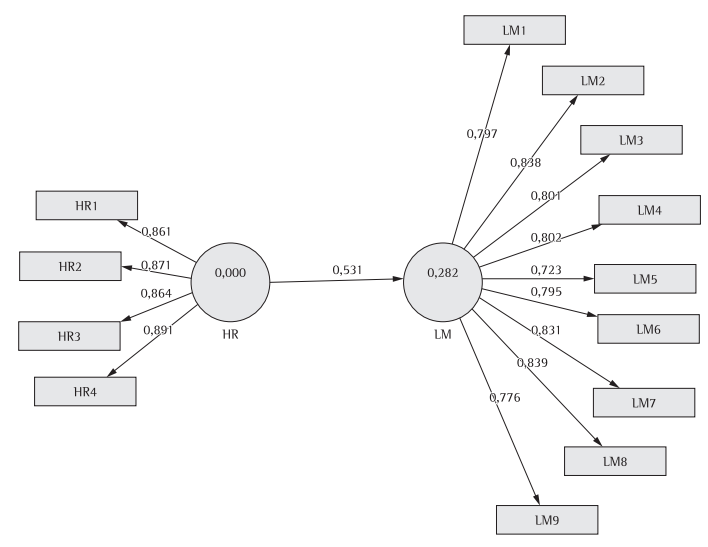

Figura 2. Modelo estrutural. 
Tabela 3. Valores de confiabilidade e de validade do modelo estrutural.

\begin{tabular}{ccccc}
\hline Construtos & $\begin{array}{c}\text { Variância média } \\
\text { extraída (AVE) }\end{array}$ & $\begin{array}{c}\text { Confiabilidade } \\
\text { composta }\end{array}$ & R Square & Alfa de Cronbach \\
\hline HR & 0,68 & 0,89 & - & 0,84 \\
LM & 0,64 & 0,94 & 0,28 & 0,68 \\
\hline
\end{tabular}

Tabela 4. Cargas cruzadas para avaliação da validade discriminante.

\begin{tabular}{lll}
\hline & HR & LM \\
\hline HR1 & 0,86 & 0,47 \\
HR2 & 0,87 & 0,48 \\
HR3 & 0,86 & 0,42 \\
HR4 & 0,69 & 0,35 \\
LM1 & 0,44 & 0,79 \\
LM2 & 0,38 & 0,83 \\
LM3 & 0,35 & 0,80 \\
LM4 & 0,38 & 0,80 \\
LM5 & 0,32 & 0,72 \\
LM6 & 0,37 & 0,79 \\
LM7 & 0,47 & 0,83 \\
LM8 & 0,51 & 0,84 \\
LM9 & 0,49 & 0,77 \\
\hline
\end{tabular}

substancial, moderado e fraco. Portanto, o efeito de HR sob LM $(0,282)$ pode ser considerado fraco-moderado.

A Tabela 3 mostra que todos os valores de confiabilidade composta são superiores a 0,7 e todos os valores de variância média extraídos são superiores a 0,5 (FOLTZ, 2008). Os coeficientes de Alfa de Cronbach e as comunalidades também são considerados adequados.

Outra medida importante para avaliar o modelo estrutural é a validade discriminante (COSTA et al., 2008). Uma das formas de garantir a validade discriminante é verificar se as variáveis realmente possuem cargas mais elevadas em seus fatores de origem. Essa análise obteve resultados adequados (Tabela 4).

Com vistas ao teste de robustez do modelo, um bootstrap de mil subamostras foi utilizado para estimar a significância estatística das relações entre as variáveis propostas e os construtos (Figura 3). Tal teste verifica se os coeficientes apresentados na Figura 2 podem ser considerados estatisticamente válidos. Segundo Hair Junior, Ringle e Sarstedt (2011), quando o teste t produzir valores próximos a 1,65, 1,96, e 2,58, o nível de significância estatística (p value) será, respectivamente, de 10\%, 5\% e 1\% (HAIR JUNIOR; RINGLE; SARSTEDT, 2011).

Todas as relações do modelo são estatisticamente válidas ao nível de significância ( $p$ value) menor ou igual a 0,01, conforme a Tabela 5 .

Por fim, cabe dizer que, de forma global, o modelo estatístico apresentou um ajuste estatístico

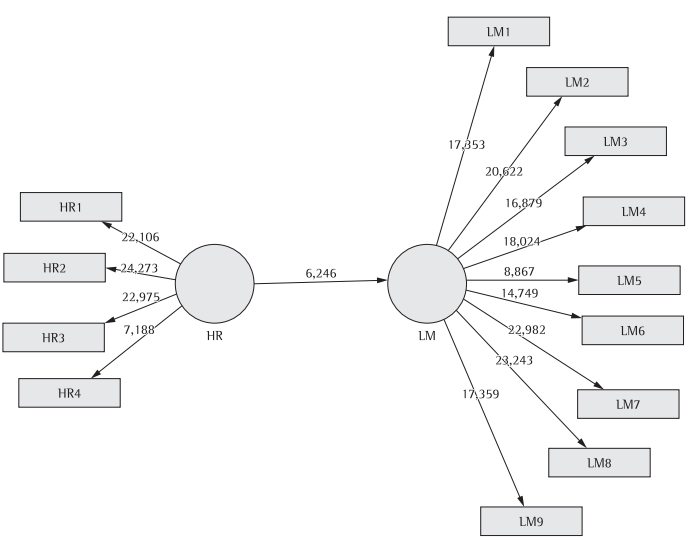

Figura 3. Modelo estrutural com bootstrapping de mil subamostras.

Tabela 5. Significância dos coeficientes dos relacionamentos do modelo.

\begin{tabular}{cccc}
\hline Relacionamentos & Coeficiente & Teste t & Significância \\
\hline LM <- HR & 0,53 & 06,24 & $*$ \\
LM1 <- LM & 0,79 & 17,35 & $*$ \\
LM2 <- LM & 0,83 & 20,62 & $*$ \\
LM3 <- LM & 0,80 & 16,87 & $*$ \\
LM4 <- LM & 0,80 & 18,02 & $*$ \\
LM5 <- LM & 0,72 & 08,86 & $*$ \\
LM6 <- LM & 0,79 & 14,74 & $*$ \\
LM7 <- LM & 0,83 & 22,98 & $*$ \\
LM8 <- LM & 0,84 & 22,24 & $*$ \\
LM9 <- LM & 0,77 & 17,35 & $*$ \\
HR1 <- HR & 0,86 & 22,10 & $*$ \\
HR2 <- HR & 0,87 & 24,27 & $*$ \\
HR3 <- HR & 0,86 & 22,97 & $*$ \\
HR4 <- HR & 0,69 & 07,18 & $*$ \\
\hline
\end{tabular}

${ }^{*} p$ value $<0,01$

considerado adequado. Para tanto, foi mensurado o Goodness-of-Fit (GoF), medida que indica o quão adequado é um modelo estatístico avaliado por modelagem de equações estruturais. Segundo Wetzels, Odekerken-Schroder e Van Oppen, (2009), um GoF adequado deve ser maior do que 0,36 . Para esta pesquisa, obteve-se um GoF adequado.

\section{Discussões e conclusões}

A proposta desta pesquisa foi verificar se a gestão de recursos humanos influencia positivamente a adoção de práticas de manufatura enxuta em empresas do 
setor automotivo brasileiro com foco no segmento de autopeças e componentes automotivos. Para tanto, elaborou-se um framework com hipótese de pesquisa, que foi testada à luz da realidade de 75 empresas do setor no Brasil.

A hipótese $\mathrm{H}_{1}$, se a gestão de recursos humanos contribui positivamente para a adoção de práticas de manufatura enxuta, foi considerada válida, revelando que, de fato, a gestão de operações requer suporte do "lado humano" das organizações. Conforme afirmam Boudreau et al. (2003), a gestão de recursos humanos é tão importante ao bom desempenho das organizações manufatureiras quanto as melhorias e atualizações tecnológicas por elas adotadas. Esta pesquisa pode, também, trazer dados empíricos do setor automotivo da realidade brasileira, em especial do setor de autopeças e componentes, confirmando os indicativos de Doolen e Hacker (2005), que discutem os aspectos positivos de recompensas e avaliação de desempenho para ações em prol da manufatura enxuta, e de Martín e García (2010), que destacam os aspectos de treinamento para melhorias na manufatura enxuta.

0 modelo estrutural proposto nesta pesquisa mostrou-se adequado, ou seja, com bom ajuste estatístico, para realizar as análises apontadas e discutidas.

A relação entre HR e LM é positiva. No entanto, o efeito de HR em LM é fraco-moderado. Nesse sentindo, outras variáveis, que não apenas ações de recursos humanos, podem influenciar a adoção de práticas de manufatura enxuta. Variáveis que eventualmente podem afetar a adoção de ações de manufatura enxuta são, por exemplo, apoio da alta administração, cultura organizacional, preocupação com a produção ambientalmente adequada, estratégia de produção etc.

Vale destacar que a amostra desta pesquisa é composta predominantemente de médias e grandes empresas e que, portanto, os seus resultados podem ajudar a entender mais a realidade de empresas com maior potencial de adotar práticas mais estruturadas de GRH e manufatura enxuta.

As limitações desta pesquisa que podem ser destacadas são: o tamanho da amostra, que apesar de todo esforço de coleta de dados chegou a apenas 75 empresas participantes, e a restrição de se analisar um único setor industrial. Além disso, como o R2 da relação entre gestão de recursos humanos e manufatura enxuta pode ser considerado fracomoderado, acredita-se que outras variáveis possam estar influenciando a adoção de práticas de manufatura enxuta nas organizações e, portanto, essas outras variáveis independentes devem ser futuramente investigadas por meio de outros frameworks.

\section{Referências}

ASSOCIAÇÃO NACIONAL DOS FABRICANTES DE VEÍCULOS AUTOMOTORES - ANFAVEA. Disponível em: <www. anfavea.org.br>. Acesso em: jun. 2011.

BHASIN, S.; BURCHER, P. Lean viewed as a philosophy. Journal of Manufacturing Technology Management, v. 17, n. 1, p. 5672, 2006. http://dx.doi.org/10.1108/17410380610639506

BIAZZO, S.; PANIZZOLO, R. The assessment of work organization in lean production: the relevance of the worker's perspective. Integrated Manufacturing Systems, v. 11, n. 1, p. 6-15, 2000. http://dx.doi. org/10.1108/09576060010303622

BORGES-ANDRADE, J. E. Desenvolvimento de medidas em avaliação de treinamento. Estudos de Psicologia, v. 7, n. especial, p. 31-43, 2002.

BOSElIE, P.; DIETZ, G.; BOON, C. Commonalities and contradictions in HRM and performance research. Human Resource Management Journal, v. 15, n. 3, p. 67-94, 2005. http://dx.doi.org/10.1111/j.1748-8583.2005.tb00154.x

BOUDREAU, J. et al. On the interface between operations and Human Resource Management. Manufacturing \& Services Operations Management, v. 5, n. 3, p. 179-202, 2003. http://dx.doi.org/10.1287/msom.5.3.179.16032

CHEN, H.; LINDEKE, R. R.; WYRICK, D. A. Lean automated manufacturing: avoiding the pitfalls to embrace the opportunities. Assembly Automation, v. 30, n. 2, p. 117-124, 2010. http://dx.doi.org/10.1108/01445151011029745

COSTA, A. C. F.; SOUZA, S. S.; SILVA, L. C. T. Investigação sobre a satisfação do usuário dos serviços prestados pelo metrô de são Paulo: um estudo exploratório, descritivo e ilustrativo com a utilização do modelo de equações estruturais. Revista de Gestão da USP, v. 15, n. especial, p. 93-108, 2008.

DAFT, R. L. Administração. 4. ed. Tradução de Fernando Gastaldo Morales. São Paulo: LTC, 1999.

DENNIS, P. Produção lean simplificada. Porto Alegre: Bookman, 2008.

DESSLER, G. Administração de recursos humanos. 2. ed. São Paulo: Prentice Hall, 2003.

DEWETTINCK, K.; REMUE, J. Contextualizing HRM in comparative research: The role of the Cranet network. Human Resource Management Review, v. 21, p. 3749, 2011. http://dx.doi.org/10.1016/j.hrmr.2010.09.010

DOOLEN, T. L.; HACKER, M. E. A review of lean assessment in organizations: an exploratory study of lean practices by electronics manufacturers. Journal of Manufacturing Systems, v. 24, n. 1, 2005. http://dx.doi.org/10.1016/ S0278-6125(05)80007-X

DOOLEN,T.L.etal.Kaizeneventsand organizationalperformance: a field study. International Journal of Productivity and Performance Management, v. 57, n. 8, p. 637-658, 2008. http://dx.doi.org/10.1108/17410400810916062

FARRIS, J. A. et al. Critical success factors for human resource in kaizen events: an empirical study. International Journal of Production Economics, v. 117, p. 42-65, 2009. http:// dx.doi.org/10.1016/j.ijpe.2008.08.051

FERGUSON, K. L.; REIO JUNIOR, T. G. Human resource management systems and firm performance. Journal of Management Development, v. 29, n. 5, p. 471-494, 2010. http://dx.doi.org/10.1108/02621711011039231

FISCHER, A. L. Um resgate conceitual e histórico dos modelos de gestão de pessoas. In: FLEURY, M. T. L. (Org.). As pessoas na organização. 9. ed. São Paulo: Gente, 2002. p. 11-33. 
FOLTZ, C. B. Why users (fail to) read computer usage policies. Industrial Management \& Data Systems, v. 108, n. 6, 70112,2008.http://dx.doi.org/10.1108/02635570810883969

FRANÇA, A. C. L.; ARELLANO, E. B. Os processos de recrutamento e seleção. In: FLEURY, M. T. L. (Org.). As pessoas na organização. 9. ed. São Paulo: Gente, 2002. p. 63-72.

HAIR JUNIOR, J. F. et al. Fundamentos de métodos de pesquisa em administração. Porto Alegre: Bookman, 2005.

HAIR JUNIOR, J. F.; RINGLE, C. M.; SARSTEDT, M. PLS-SEM: Indeed a silver bullet. Journal of Marketing Theory and Practice, v. 19, n. 2, p. 139-151, 2011. http://dx.doi. org/10.2753/MTP1069-6679190202

JANSSENS, M.; STEYAERT, C. HRM and Performance: A plea for reflexivity in HRM studies. Journal of Management Studies, v. 46, n. 1, p. 143-155, 2009. http://dx.doi. org/10.1111/j.1467-6486.2008.00812.x

LENGNICK-HALL, M. L. et al. Strategic human resource management: The evolution of the field. Human Resource Management Review, v. 19, n. 2, p. 64-85, 2009. http:// dx.doi.org/10.1016/j.hrmr.2009.01.002

MARTÍN, T. B.; GARCíA, J. A. M. Producción ajustada y recursos humanos: resultados sobre la efectividad empresarial. Revista Europea de Direccion y Economia de La Empresa, v. 19, n. 4, p. 117-134, 2010.

MENEZES, L. M.; WOOD, S.; GELADE, G. The integration of human resource and operation management practices and its link with performance: a longitudinal latent class study. Journal of Operations Management, v. 28, p. 455471, 2010. http://dx.doi.org/10.1016/j.jom.2010.01.002

MILKOVICH, G. T.; BOUDREAU, J. W. Administração de recursos humanos. São Paulo: Atlas, 2006.

MURILLO-LUNA, J. L.; GARCÉS-AYERBE, C.; RIVERA-TORRES, $P$. Barriers to the adoption of proactive environmental strategies. Journal of Cleaner Production, v. 19, p. 14171425, 2011. http://dx.doi.org/10.1016/j.jclepro.2011.05.005

OHNO, T. Toyota production system: beyond large scale production. Cambrigde: Productivity Press, 1988.

OLIVEIRA, P. W. S.; LEONE, R. G. J. Gestão estratégica de benefícios: proposição de um modelo matemático para assistência médica dos funcionários. RAM - Revista de Administração Mackenzie, v. 9, n. 2, p. 104-127, 2008.

PAAUWE, J. HRM and performance: achievements, methodological issues and prospects. Journal of Management Studies, v. 46, n. 1, 2009. http://dx.doi. $\operatorname{org} / 10.1111 /$ j.1467-6486.2008.00809.x
PETTERSEN, J. Defining lean production: some conceptual and practical issues. The TQM Journal, v. 21, n. 2 , p. 127-142, 2009. http://dx.doi. org/10.1108/17542730910938137

SCHERRER-RATHJE, M.; BOYLE, T. A.; DEFLORIN, P. Lean, take two! Reflections from the second attempt at lean implementation. Business Horizons, v. 52, p. 79-88, 2009. http://dx.doi.org/10.1016/j.bushor.2008.08.004

SCHONBERGER, R. J. Japanese production management: an evolution - with mixed success. Journal of Operations Management, v. 25, p. 403-419, 2007. http://dx.doi. org/10.1016/j.jom.2006.04.003

SCHULER, R. S.; JACKSON, S. E. Linking competitive strategies with human resource practices. Academy of Management Executive, v. 1, n. 3, p. 207-220, 1987. http://dx.doi. org/10.5465/AME. 1987.4275740

SHAH, R.; WARD, P. T. Lean manufacturing: context, practice bundles, and performance. Journal of Operations Management, v. 21, p. 129-149, 2003. http://dx.doi. org/10.1016/S0272-6963(02)00108-0

SHAH, R.; WARD, P. T. Defining and developing measures of lean production. Journal of Operations Management, v. 25 , p. $785-805$, 2007. http://dx.doi.org/10.1016/j. jom.2007.01.019

SOSIK, J. J.; KAHAl, S. S.; PIOVOSO, M. J. Silver bullet or voodoo statistics?: A primer for using least squares data analytic technique in group and organization research. Group \& Organization Management, v. 35, n. 5, p. 5-36, 2009. http://dx.doi.org/10.1177/1059601108329198

STONER, J. A. F.; FREEMAN, R. E. Administração. 5. ed. tradução de Alice Alves Calado. São Paulo: LTC, 1999.

SYNODINOS, N. E. The "art" of questionnaire construction: some important considerations for manufacturing studies. Integrated Manufacturing Systems, v. 14, n. 3, p. 221237,2003.http://dx.doi.org/10.1108/09576060310463172

TÜRK, K.; ROOLAHT, T. Appraisal and compensation of the academic staff in Estonian public and private universities: a comparative analysis. Trames, v. 11, n. 2, p. 206-222, 2007.

WETZELS, M.; ODEKERKEN-SCHRODER, G.; VAN OPPEN, C. Using PLS path modeling for assessing hierarchical construct models: guidelines and empirical illustration. MIS Quartely, v. 33, n. 1, p. 177-195, Mar 2009.

WOMACK, J. P.; JONES, D. T.; ROOS, D. The machine that changed the world. New York: Rawson Associates, 1990.

\section{Human resource management and lean manufacturing: empirical evidence from the Brazilian automotive sector}

Abstract

The aim of this paper is to verify whether the practices of human resource management have a positive influence on the adoption of lean manufacturing practices in Brazilian companies in the automotive sector. To this aim, we proposed a framework linking these concepts to the proposed research hypothesis. Data collected from 75 companies were analyzed using structural equation modeling. The research demonstrates that human resource management indeed tends to influence the adoption of lean manufacturing practices in a weak-moderate manner.

\section{Keywords}

Human resource management. Lean manufacturing. Automotive sector. Brazil. 
Apêndice A. Assinale a alternativa que corresponde ao nível de qualidade das práticas de recursos humanos:

\begin{tabular}{|l|l|l|l|l|l|}
\hline & $\begin{array}{c}\text { Discordo } \\
\text { totalmente }\end{array}$ & $\begin{array}{c}\text { Discordo } \\
\text { (parcialmente) }\end{array}$ & $\begin{array}{c}\text { Nem discordo, } \\
\text { nem concordo }\end{array}$ & $\begin{array}{c}\text { Concordo } \\
\text { (parcialmente) }\end{array}$ & $\begin{array}{c}\text { Concordo } \\
\text { totalmente }\end{array}$ \\
\hline $\begin{array}{l}\text { HR1 - O processo de recrutamento e } \\
\text { seleção é eficiente e eficaz }\end{array}$ & & & & & \\
\hline $\begin{array}{l}\text { HR2 - O processo de treinamento é } \\
\text { eficiente e eficaz }\end{array}$ & & & & & \\
\hline $\begin{array}{l}\text { HR3 - O sistema de avaliação de } \\
\text { desempenho do funcionário é eficiente } \\
\text { e eficaz }\end{array}$ & & & & & \\
\hline $\begin{array}{l}\text { HR4 - O sistema de recompensas } \\
\text { (salário e comissão) é eficiente e eficaz }\end{array}$ & & & & & \\
\hline $\begin{array}{l}\text { HR5 - O sistema de benefícios } \\
\text { (saúde, lazer) é eficiente e eficaz }\end{array}$ & & & & & \\
\hline
\end{tabular}

Apêndice B. Assinale qual é o nível de implementação, na sua empresa, das práticas de manufatura enxuta listadas na primeira coluna:

\begin{tabular}{|l|l|l|l|l|l|}
\hline & $\begin{array}{c}\text { Não } \\
\text { implementado }\end{array}$ & $\begin{array}{c}\text { Começando a } \\
\text { implementar }\end{array}$ & $\begin{array}{c}\text { Parcialmente } \\
\text { implementado }\end{array}$ & $\begin{array}{c}\text { Consideravelmente } \\
\text { implementado }\end{array}$ & $\begin{array}{c}\text { Completamente } \\
\text { implementado }\end{array}$ \\
\hline $\begin{array}{l}\text { LM1 - Envolvimento do trabalhador } \\
\text { no processo produtivo }\end{array}$ & & & & & \\
\hline $\begin{array}{l}\text { LM2 - Busca sistemática da melhoria } \\
\text { contínua na produção }\end{array}$ & & & & & \\
\hline $\begin{array}{l}\text { LM3 - 5S (separar/organizar/limpar/ } \\
\text { padronizar/manter) }\end{array}$ & & & & & \\
\hline $\begin{array}{l}\text { LM4 - Manutenção produtiva total } \\
\text { dos equipamentos }\end{array}$ & & & & & \\
\hline LM5 - Kanban (sistema puxado) & & & & & \\
\hline $\begin{array}{l}\text { LM6 - Just-in-time (busca manter o } \\
\text { fluxo contínuo) }\end{array}$ & & & & & \\
\hline LM7 - Redução de estoque & & & & & \\
\hline $\begin{array}{l}\text { LM8 - Círculo kaizen (grupos de } \\
\text { discussão para melhorar processos) }\end{array}$ & & & & & \\
\hline LM9 - Colaboração com fornecedores & & & & & \\
\hline
\end{tabular}

Apêndice C. Outras medidas estatísticas do construto HR

Média e desvio padrão das variáveis do construto $\mathrm{HR}$

\begin{tabular}{|c|c|c|}
\hline Variáveis & Média & Desvio padrão \\
\hline HR1 & 3,48 & 1,08 \\
\hline HR2 & 3,52 & 1,10 \\
\hline HR3 & 3,20 & 1,30 \\
\hline HR4 & 2,34 & 1,12 \\
\hline
\end{tabular}

Correlação de Pearson para as variáveis do construto HR

\begin{tabular}{|c|c|c|c|c|}
\hline & HR1 & HR2 & HR3 & HR4 \\
\hline HR1 & 1 & & & \\
\hline HR2 & $0,71^{*}$ & 1 & & \\
\hline HR3 & $0,58^{*}$ & $0,72^{*}$ & 1 & \\
\hline HR4 & $0,49^{*}$ & $0,35^{*}$ & $0,55^{*}$ & 1 \\
\hline
\end{tabular}

${ }^{*} p$ value $<0,05$ 
Freitas, W. R. S. et al.

Gestão de recursos humanos ... setor automotivo brasileiro. Production, v. 24, n. 2, p. 451-461, Apr./June 2014

Apêndice D. Outras medidas estatísticas do construto LM

Média e desvio padrão das variáveis do construto LM

\begin{tabular}{|c|c|c|}
\hline Variáveis & Média & Desvio padrão \\
\hline LM1 & 3,69 & 1,12 \\
\hline LM2 & 3,86 & 1,05 \\
\hline LM3 & 3,78 & 1,18 \\
\hline LM4 & 3,20 & 1,27 \\
\hline LM5 & 2,90 & 1,41 \\
\hline LM6 & 3,04 & 1,42 \\
\hline LM7 & 3,52 & 1,01 \\
\hline LM8 & 3,20 & 1,37 \\
\hline LM9 & 3,17 & 1,18 \\
\hline
\end{tabular}

Correlação de Pearson para as variáveis do construto LM

\begin{tabular}{|l|c|c|c|c|c|c|c|c|c|}
\hline & LM1 & LM2 & LM3 & LM4 & LM5 & LM6 & LM7 & LM8 & LM9 \\
\hline LM1 & 1 & & & & & & & & \\
\hline LM2 & $0,737^{*}$ & 1 & & & & & & & \\
\hline LM3 & $0,637^{*}$ & $0,708^{*}$ & 1 & & & & & & \\
\hline LM4 & $0,627^{*}$ & $0,653^{*}$ & $0,671^{*}$ & 1 & & & & & \\
\hline LM5 & $0,499^{*}$ & $0,479^{*}$ & $0,518^{*}$ & $0,535^{*}$ & 1 & & & & \\
\hline LM6 & $0,512^{*}$ & $0,568^{*}$ & $0,546^{*}$ & $0,657^{*}$ & $0,771^{*}$ & 1 & & & \\
\hline LM7 & $0,600^{*}$ & $0,643^{*}$ & $0,528^{*}$ & $0,534^{*}$ & $0,615^{*}$ & $0,701^{*}$ & 1 & & \\
\hline LM8 & $0,598^{*}$ & $0,688^{*}$ & $0,638^{*}$ & $0,579^{*}$ & $0,530^{*}$ & $0,581^{*}$ & $0,677^{*}$ & 1 & \\
\hline LM9 & $0,524^{*}$ & $0,567^{*}$ & $0,590^{*}$ & $0,592^{*}$ & $0,419^{*}$ & $0,497^{*}$ & $0,627^{*}$ & $0,647^{*}$ & 1 \\
\hline
\end{tabular}

*p value $<0,05$. 